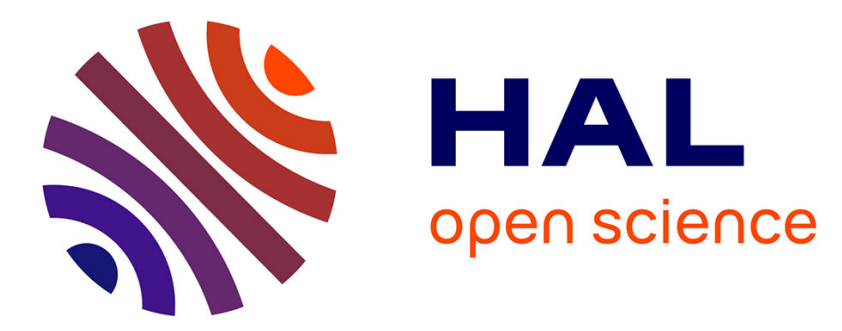

\title{
Large scale structure and dynamics of polysaccharide gels
}

\author{
A. Deriu, F. Cavatorta, D. Di Cola, H. Middendorf
}

\section{To cite this version:}

A. Deriu, F. Cavatorta, D. Di Cola, H. Middendorf. Large scale structure and dynamics of polysaccharide gels. Journal de Physique IV Proceedings, 1993, 03 (C1), pp.C1-237-C1-247. 10.1051/jp4:1993120 . jpa-00251563

\section{HAL Id: jpa-00251563 https://hal.science/jpa-00251563}

Submitted on 1 Jan 1993

HAL is a multi-disciplinary open access archive for the deposit and dissemination of scientific research documents, whether they are published or not. The documents may come from teaching and research institutions in France or abroad, or from public or private research centers.
L'archive ouverte pluridisciplinaire HAL, est destinée au dépôt et à la diffusion de documents scientifiques de niveau recherche, publiés ou non, émanant des établissements d'enseignement et de recherche français ou étrangers, des laboratoires publics ou privés. 


\title{
Large scale structure and dynamics of polysaccharide gels
}

\author{
A. DERIU, F. CAVATORTA, D. DI COLA and H.D. MIDDENDORF \\ Dipartimento di Fisica, Università di Parma, Italy \\ * Clarendon Laboratory, University of Oxford, U.K.
}

\begin{abstract}
We review some theoretical and experimental aspects of the application of the scattering of neutrons and of Mössbauer radiation to the study of the structure and molecular dynamics of polysaccharide gels. The results demonstrate clearly that one can obtain detailed information on the polysaccharide network over distances up to about 1000 $\AA$, and on the molecular dynamics of the polysaccharide backbone and of the associated hydration water.
\end{abstract}

Résumé Nous présentons une revue des aspects théoriques et expérimentaux de l'application de la diffusion des neutrons et de la radiation Mössbauer à l'étude de la structure et dynamique moleculaire des gels de polysaccharides. Nos résultats prouvent que on peut avoir des informations détaillées sur la structure à grande échelle $(\sim 1000 \AA)$ et sur la dynamique moléculaire du réseau biopolymerique et de l'eau d'hydratation.

\section{INTRODUCTION}

During the 1980s the study of aqueous (bio)polymer gels moved into the foreground of research in physical chemistry and biophysics, after many years of relative neglect. This was due to a variety of reasons. First of all, as regards complexity, these gels are intermediate between dilute solutions and crystalline or paracrystalline systems, and they offer therefore much scope for investigating basic aspects of water-biopolymer interactions that mimic those in biological tissues. Secondly, some aqueous gels are used very extensively in biochemistry and molecular biology to separate nucleic acids and proteins by electrophoresis. Thirdly, such gels are finding increasing applications in medicine, both as pharmaceutical products (ophthalmology, controlled drug release) and as components of biocompatible materials. Finally, there are several little developed but potentially important applications such as biosensors, muscle models, etc. Reviews on both fundamental aspects and applications of polymer gels have been published recently (1).

From the viewpoint of molecular physicist, gels are large-scale two-component or three-component systems that pose a number of challenging fundamental problems. On account of their random network structure, phase diagrams, rehological behaviour and transport properties, they offer many opportunities for testing and extending modern concepts of fractality, percolation and reptation processes. The application of such concepts to complex polymer systems is facilitated by the fact that aqueous gels are common tools in present-day molecular biology and bioengineering, This has provided important links to application-oriented experimental work and to computer simulation studies of large molecular assemblies. 
Despite the extensive use of aqueous (bio)polymer gels in the biosciences, the molecular structure and dynamics of commonly used gels remain relatively unexplored. Much impetus for more extensive research in this area has come from the exploitation of new or improved physical techniques, in particular radiation scattering techniques. Further optimisation of gel technologies depends critically on a detailed knowledge of the microscopic structure of gels and a good understanding of the dynamics of their interaction with water and with 'guest' molecules.

\section{APPLICATION OF RADIATION SCATTERING TECHNIQUES}

In the sections to follow, we review recent experimental work performed on polysaccharide gels using radiation scattering techniques, and discuss the interpretation of static and dynamic structure factors $S(Q)$ and $S(Q, \omega)$ in the framework of analytical models as well as computer simulation results.

The case for developing the application of radiation scattering techniques to polysaccharide gels rests on the following points:

(i) From the classical X-ray work of Arnott, Rees et al. (2), together with some spectroscopic information at optical frequencies, supplemented also by computer simulations (3), we know in atomic detail the primary and secondary structure of slightly hydrated polysaccharide fibres (mainly agarose, carrageenans, hyaluronates). Our knowledge of the gel state proper, on the other hand, is limited to results from techniques probing distances $d>3000 \AA$ and/or motions with frequencies $<10 \mathrm{MHz}(4-6)$. To understand qualitatively how the remarkable electrophoretic and rheological behaviour of many gels is determined by their microscopic properties, it is essential to obtain data linking these two space-time domains, and to cover in particular the transition region from long-range diffusive processes to more localised interactions at the biopolymer-water interfaces.

(ii) The interpretation of structure factors $S(Q)$ and $S(Q, \omega)$, is greatly facilitated by extensive theoretical $(7,8)$ and experimental $(9,10)$ work on polymer networks and chain molecule dynamics. Moreover gels make perfect samples for radiation scattering experiments. Slab samples of 0.3 to 2 $\mathrm{mm}$ thickness can be produced easily and maintained under a variety of conditions (electric fields, mechanical stresses, secondary irradiation, etc.). For neutron scattering, it is possible to create a wide range of hydrogen/deuterium contrast by exchange of buffers.

\section{LARGE-SCALE STRUCTURE}

The double-stranded helical structure of polysaccharide fibres, first established for i-carrageenan by Anderson et al. (11), is common to many polysaccharides forming aqueous thermoreversible gels. Certain polysaccharides, however, give gels consisting of triple helices, or polymerise only by forming inclusion complexes with ions (the alginates).

The building block of agarose, the most widely used and extensively studied polysaccharide, is a dimer made up of $(1 \rightarrow 4)$ linked 3,6 anhydro $\alpha$-L galactose bridged to a $(1 \rightarrow 3)$ linked $\beta$-D galactose by a glycosidic link (Fig. 1). Upon gelling, the agarose chains aggregate giving a random $3 \mathrm{D}$ network of bundles of double helices connected through junction zones. The classical X-ray work on hydrated fibers and films of polysaccharides of the agar-carrageenan family has established atomic details of the primary and secondary structure and has, in conjunction with optical data, provided evidence for chain association and network formation in gels $(2,12)$. The double helix of agarose, in particular, has left-handed threefold symmetry with a $19 \AA$ pitch $(2,12)$. Interchain association occurs by lateral aggregation through hydrogen-bonding, i.e. by 'physical' cross-linking (Fig. 1). 
The large-scale structure of the network in the gel state proper is still poorly understood. The presence of non-covalent cross-links complicates the physical description of the network properties considerably, since the number and position of the cross-links can fluctuate with time and temperature. Moreover, any equilibrium configuration is likely to be characterised by a rather broad polydispersity of the chain bundles (with respect to both length and thickness) and also by the extent and spatial distribution of the junction zones as well (13).

Detailed information on the long range structure of gels is of great relevance for a deeper understanding of the gelation mechanisms on which there are diverging opinions in the literature (14). Structural data over a wide range of scale length are needed to interpret the macroscopic viscoelastic and rheological properties and to provide a basis for studying electrophoretic processes.

a)

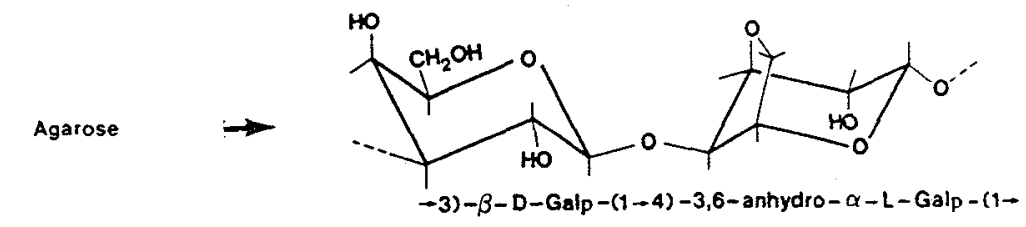

b)
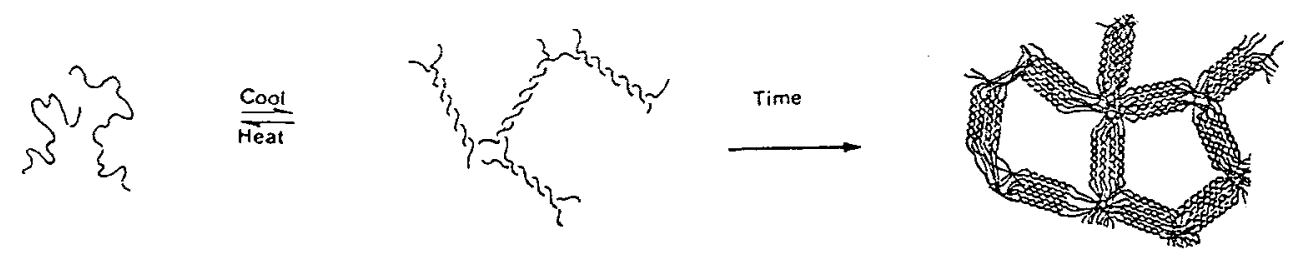

Fig. 1 a) The agarose dimer. b) Sketch of the process leading to the hierarchical structure of agarose gels.

Various attempts have been made to define gel structures in terms of few simple parameters such as the branching fraction (15) or correlation length (8). Inevitably these theories give an oversimplified picture of gel structure, which is useful as a zero-order approximation to the description of such complex molecular arrangements. The universal properties which emerge from these models are often quantified in terms of scaling laws in the framework of fractal concepts. Low spatial resolution diffraction experiments as small angle scattering of X-rays (SAXS), neutrons (SANS), and light scattering (LS) can elucidate the gel structure by providing information about the molecular arrangement over distances from $\sim 20 \AA$ up to a few $1000 \AA$.

Using SANS instruments at the high flux reactor of the Institut Laue-Langevin (ILL), Grenoble (D17 and D11 diffractometers) and at the ISIS pulsed neutron facility of the Rutherford Appleton Laboratory, near Oxford (LOQ diffractometer), we have started to build up a database of diffraction patterns $S(Q)$ on agarose gels as a function of concentration, $\mathrm{H} / \mathrm{D}$ contrast, and temperature. Data collected so far are mainly from fully $\mathrm{D}_{2} \mathrm{O}$-exchanged gels in a range of scattering vectors $Q$ from 0.003 to $0.2 \AA^{-1}$, and with concentrations $\mathrm{C}$ from 0.01 to $0.5(\mathrm{C}=\mathrm{g}$ agarose/g water $)(16,17)$. A typical diffraction pattern is shown in a log-log representation in Fig. 2. Different characteristic regions are apparent in this plot. At very low $Q\left(Q \leq 0.005 \AA^{-1}\right)$ and low concentrations $\mathrm{C}$ $(\leq 0.01)$ we observe a Guinier behaviour characteristic of a distribution of long thin rods. At higher concentrations the curves slope upwards, indicating that the thin rod approximation (i.e. negligible radius of gyration of the bundle cross section with respect to the average distance between cross-links) no longer holds for more concentrated gels. In the regions where $0.005 \leq Q \leq 0.009$ $\AA^{-1}$ (region I) and $0.009 \leq Q \leq 0.075 \AA^{-1}$ (region II), we find linear segments from which it is possible to derive scaling parameters which relate to the exponent $\mu$ in $S(Q) \propto Q^{-\mu}$. At $Q \geq 0.075$ 
$\AA^{-1}$, there is a change in slope again, accompanied by intensity changes which reflect structural features with scale lengths below $100 \AA$. The value of $\mu$ derived in region $I$ is almost concentration independent and close to 1.0, while in region II it varies from about 2.2 to 1.0 as $\mathrm{C}$ increases from 0.01 to 0.4 . It can be related to scaling parameters which reflect both the (limited) fractal structure of such gels and their inherent polydispersity.

For an ideal monodisperse gel the scattering intensity can be expressed in terms of the correlation length $\xi$ (i.e. the size of non-correlated domains in the gel phase) and the fractal dimension $D$ inside the domains as (18):

$$
S(Q) \propto \frac{\xi^{2}}{\left(1+(D+1) \xi^{2} Q^{2} / 3\right)^{D / 2}}
$$

For an improved model taking into account the mass polydispersity of the domains quantified by a power law with exponent $\tau$, the scattered intensity reduces asymptotically to:

$$
\begin{aligned}
& S(Q) \propto Q^{-D(3-\tau)}, \quad(\xi Q<<1) \\
& S(Q) \propto Q^{-D}, \quad(\xi Q>>1)
\end{aligned}
$$

Percolation cluster theories (19) predict $\tau=2.2$ and $D=2.5$ near the gelation point, leading to a polydispersity-smeared fractal dimension $\mu=D(3-\tau)=2.0$ i.e. close to what we obtain for low concentration gels. The variation of the observed $\mu$ with concentration might be attributed to values of the mass distribution exponent $\tau$ different (probably smaller) from that of simple percolation models. This could result from the multiple step process formation of cross-linking domains as sketched in Fig. 1. We must note however that scaling laws for $S(Q)$ with analogous values of $\mu(\mu=2)$ are also predicted by other theoretical approaches to the gelation process (20), and the interpretation of the SANS results in terms of fractal models cannot therefore be considered as univocal.

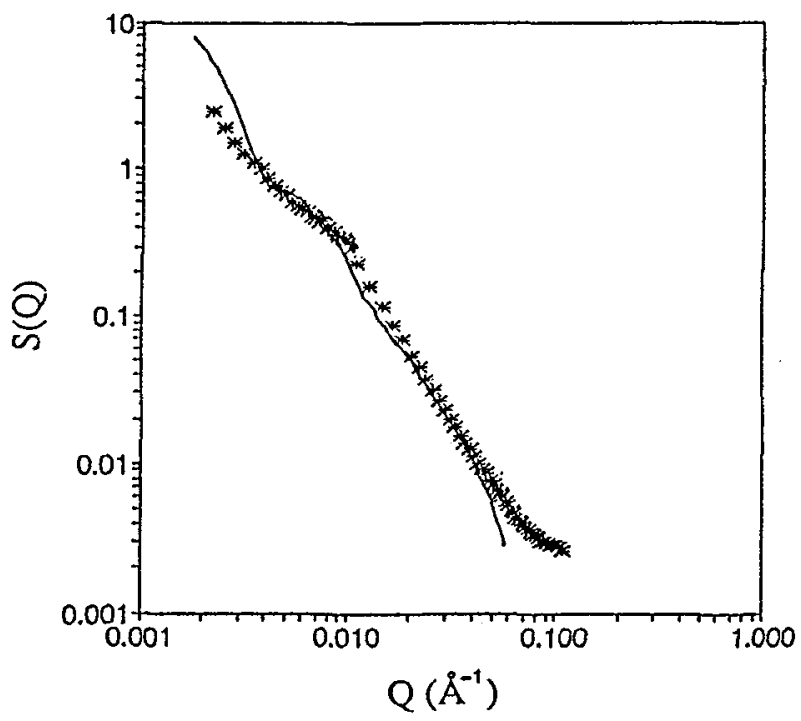

Fig. 2 log-log plot of the intensity scattered by a set of fully $\mathrm{D}_{2} \mathrm{O}$ exchanged gel samples with concentration $C=0.01$. The asterisks are the experimental data, and the continuous line is the result of a simulation described in the text. 
A complementary approach to the interpretation of SANS diffraction data can come from computer simulations of model gels. The well-known Debye method of calculating $S(Q)$ for biomolecular assemblies in solution has recently been extended to network structures. This approach is based on the well-known method of calculating $S(Q)$ for biomolecules in solution by way of model density distributions consisting of assemblies of spherically symmetric scattering elements. This method has been used very successfully already for SANS from glycoproteins by Perkins et al. (21). We first create a 3D 'skeleton' network by a quasi-random algorithm mimicking the gelation process. Each line connecting two nodes then becomes the axis of a 'rod', i.e. a subassembly of scattering elements with overall cylindrical or helical symmetry. Specific rod structures modelling e.g. the density distribution of bundles of polysaccharide fibres are generated by subroutines, with the possibility of varying rod diameters either randomly or correlated with rod length. Realistic gel models generated in this way consist of $>10^{5}$ scattering elements, and the task of calculating $S(Q)$ via Debye's formula becomes non trivial computationally. Initial results for 12 smaller model gels $\left(\sim 3 \times 10^{4}\right.$ elements each) have been most encouraging and will be presented at a forthcoming conference (22). We plan to run larger models, varying concentration and rod structures, with the aim of obtaining $S(Q)$ patterns that can be compared with our SANS measurements. A first comparison between experimental and simulated $S(Q)$ obtained by averaging the patterns from the 12 model gels mentioned above is shown in Fig. 2.

\section{MOLECULAR DYNAMICS}

The translational and rotational mobilities of water trapped by reticulate structures and porous solids are known to be altered substantially relative to those of bulk water. Detailed computer simulation studies on agarose hydration (3) have shown that agarose chains, like most other biopolymers, have specific binding sites for water, such that the first hydration shell around the double helices is made up from $\mathrm{H}_{2} \mathrm{O}$ molecules which are irrotationally bound (primary hydration water), and their dynamics is expected to follow closely that of the polymeric skeleton of agarose. In dilute gels, pictured as a 'molecular scaffolding' of hydrophilic fibres with relatively large mesh sizes, the network through its van der Waals volume and $\mathrm{H}$-bonding moieties slows down the diffusive motions of vicinal water layers over 10-15 $\AA$. It is still controversial to what extent segmental polymer motions or highly damped collective excitations of the network give rise to additional 'dynamic structuring', and thus influence the rapid formation and breaking of H-bonded water clusters further away from the fibre surfaces. This is a fundamental question that is central to current disputes about the special properties of water in cells and tissues (23).

For a full characterisation of the water and polysaccharide chain dynamics one has to cover a large interval of characteristic times from motions in the picosecond region down to a few 100 nanoseconds. Various spectroscopic techniques can be applied jointly to cover such time domain. Here we will be mainly concerned with results obtained by Rayleigh Scattering of Mössbauer Radiation (RSMR), quasi-elastic (QENS) and inelastic (INS) neutron scattering. By combining results from these techniques we are able to cover a time interval from $10^{-7}$ to $10^{-13} \mathrm{~s}$.

\section{RSMR results}

The RSMR technique makes use of $\gamma$-rays $(\lambda \sim 1 \AA)$ emitted by a Mössbauer source. The radiation undergoes Rayleigh-Thompson scattering from the electrons in the sample and is then energy analysed by an appropriate Mössbauer resonant absorber (24). The main feature of RSMR is its capability of detecting variations in the energy of the diffracted photons comparable with the typical widths of the Mössbauer lines $\left(\Gamma \simeq 10^{-9}-10^{-10} \mathrm{eV}\right.$ ). The setup of an RSMR experiment is similar to that of a common $\mathrm{X}$-ray diffraction apparatus: a high activity Mössbauer source is used 
in place of the X-ray generator, and the Mössbauer resonant absorber is placed in the diffracted beam between the sample and the detector. This will resonantly absorb only photons which have undergone scattering with energy variation lower than its Mössbauer linewidth $\Gamma$. Scattering events with energy changes larger than $\Gamma$ are considered as inelastic. Therefore, by combining measurements with and without resonant absorber, it is possible to determine the elastic and total inelastic structure factors according to:

$$
\begin{gathered}
S_{e l}(\mathbf{Q})=\int_{-\Gamma}^{+\Gamma} S(\mathbf{Q}, \omega) d \omega \\
S_{i n}(\mathbf{Q})=S(\mathbf{Q})-S_{e l}(\mathbf{Q})
\end{gathered}
$$

By use of RSMR $S_{e l}$ and $S_{\text {in }}$ were measured in agarose gel samples at room temperature in a large range of concentrations $\mathrm{C}$ from 0.2 to a totally dried gel sample (obtained by heating at $\sim 80^{\circ} \mathrm{C}$ a sample kept under moderate vacuum) (25). The very low concentration region could not be covered by RSMR because of the dominating background signal from the mobile interstitial water which caused $S_{e l}$ to drop down to immeasurably small values.

The dependence of the fraction of elastic scattering $f\left(f=S_{e l}(Q) / S(Q)\right)$ upon the percentage water content $\delta(\delta=100 /(1+C))$ is shown in Fig. 3 for three selected values of $Q$.

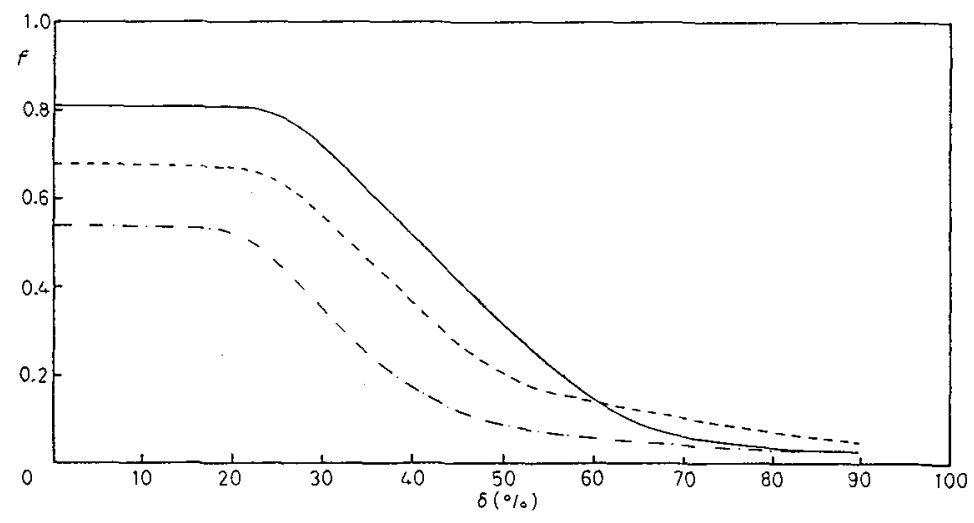

Fig. 3. Dependence of the elastic fraction $f$ on percentage water content $\delta$ for hydrated agarose gels at room temperature and at different $Q$ values. $Q=1.34,1.78,2.66 \AA^{-1}$ (top to bottom).

It is seen that starting from the dry state, water uptake in isothermal conditions up to $\delta \sim$ $25 \%(\mathrm{C} \sim 3.0)$ does not modify the dynamics of the system. Moreover the dynamics of water molecules closely associated with the polysaccharide chains is essentially the same as that of the polymer backbone. It is worth noting that this hydration level corresponds to an almost full first layer of hydration ( $\sim 5.7$ water molecules per agarose unit). Further addition of water increases the mobility of both the saccharide chains and the associated water. In the frame of a quasi-harmonic approximation it is possible to derive mean square atomic displacements $\left\langle u^{2}\right\rangle$ by expressing $f$ as:

$$
f=\gamma e^{-\frac{1}{2} Q^{2}\left\langle u^{2}\right\rangle}
$$

where $\gamma=\mathrm{I}_{\text {coh }} /\left(\mathrm{I}_{\text {coh }}+\mathrm{I}_{\text {Compton }}\right)$ is the ratio of the coherent to total scattering. 
The $\left\langle u^{2}\right\rangle$ values derived from the $f$ data of Fig. 3 are displayed in Fig. 4 .

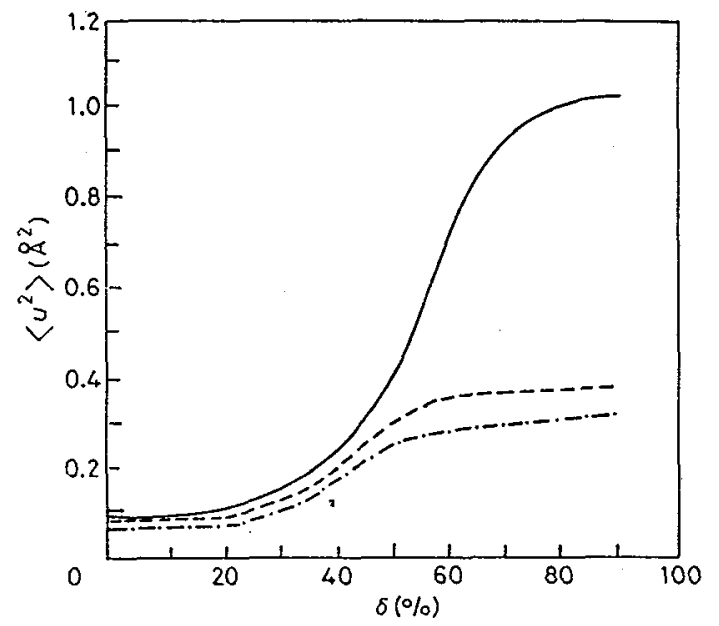

Fig. 4. Mean square atomic displacements $\left\langle u^{2}\right\rangle$ deduced from the data of Fig. 3. The three curves are for $Q=1.34,1.78,2.66 \AA^{-1}$ (top to bottom).

\section{QENS results}

The picture emerging from various results of a distribution of water molecules with different mobility is confirmed by quasi-elastic neutron scattering results. These were obtained using time-offlight (TOF) and backscattering spectrometers at the ILL (Grenoble), and at the pulsed neutron source ISIS (Oxford). In this way it was possible to cover a sufficiently large region of energy transfer $\hbar \omega$ providing information on both the relatively slow dynamics of the backbone, and that of associated water of hydration. QENS spectra in hydrogenous gels are dominated by proton incoherent dynamic structure factors $S_{i n c}(Q, \omega)$ because of the very large $n-p$ incoherent crosssection $\left(80 \times 10^{-24} \mathrm{~cm}^{2}\right)$. In order to take into account the different contributions from proton species with different mobilities present in the gel, we have considered an overall proton incoherent structure factor made up of three contributions:

$$
S(Q, \omega)=f^{I} W^{I} \delta(\omega)+f^{I I} W^{I I} S_{\text {rot }}(Q, \omega)+f^{I I I} W^{I I I} \cdot\left[S_{\text {trans }}(Q, \omega) \otimes S_{\text {rot }}(Q, \omega)\right]
$$

The population factors (satisfying $f^{I}+f^{I I}+f^{I I I}=1$ ) refer to:

(I) a first shell of hydration of irrotationally bound protons plus those of the polymer backbone;

(II) protons capable of performing hindered rotational motions, but site-bound up to times $\sim$ $10^{-8} \mathrm{~s}$;

(III) protons belonging to the interstitial aqueous phases;

Here $W^{i}$ are the respective Debye-Waller factors. The dynamics of the first proton population $(I)$ has been investigated using IN10 at the ILL (17). The spectra can be separated into a central slightly broadened peak superimposed on a broader component reflecting mainly water translational and rotational modes. Fig. 5 shows a $\log$-log plot of the quasi-elastic difference widths of the central peaks with respect to those measured for a frozen sample $(T=266 \mathrm{~K})$ assumed to be purely elastic. The data show the existence of two distinct regions with a crossover at $Q \sim 0.5$ $\AA^{-1}$; this dependence can be interpreted in terms of current theories of the dynamics of polymeric 
chains in solution (26).

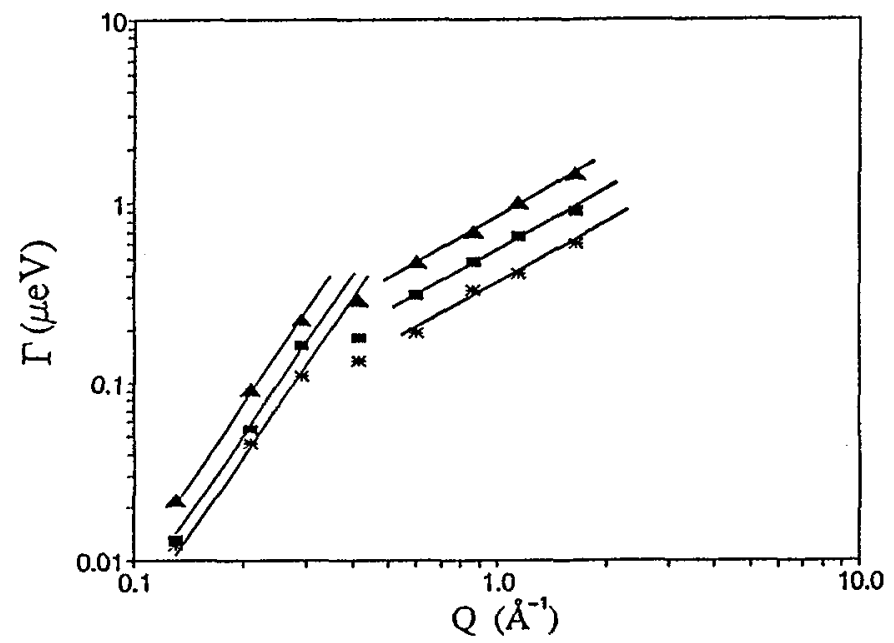

Fig. 5 log-log plots of the linewidth $\Gamma$ (HWHM) of the quasi-elastic spectrum vs. $Q$ for a fully hydrogenous gel with agarose concentration 0.075 at different temperatures: $(*) T=283$, (D) $T=303 \mathrm{~K},(\mathbf{A}) T=326 \mathrm{~K}$. The continous lines are linear fits to the low $Q$ data $\left(\sim Q^{3}\right)$ and high- $Q$ data $(\sim Q)$. The resulting values for $\Gamma / \hbar Q^{3}$ in $\mathrm{cm}^{3} / \mathrm{s}$ are: $0.72 \times 10^{-13}(T=283 \mathrm{~K})$, $0.96 \times 10^{-13}(T=303 \mathrm{~K}), 1.44 \times 10^{-13}(T=326 \mathrm{~K})$.

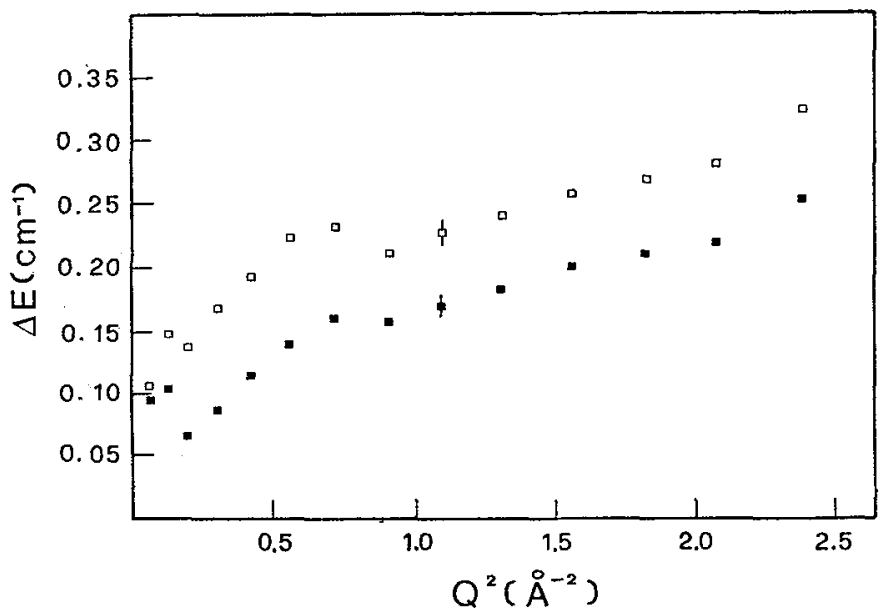

Fig. 6 Difference halfwidths $\Delta \mathrm{E}$ (FWHM) vs. $Q^{2}$ of the quasi-elastic peaks relative to a vanadium scattering standard. The data are for an hydrogenous gel with concentration $1.0 \mathrm{~g} / \mathrm{cm}^{3}$. The two curves are for $T=296 \mathrm{~K}(\mathbf{D})$, and $T=337 \mathrm{~K}(\mathrm{\square})$.

The broader component which originates from the contributions of type- $I I$ and type-III protons has a width which markedly depends upon gel concentration and temperature as shown in Fig. 6. Initial results on this component have been reported $(27,28)$, and a second more detailed paper has been submitted (29). In the latter a detailed analysis the temperature and concentration dependence of the diffusive parameters derived from spectra is given, and the principal conclusions are the following: 
(1) the dynamic properties of water contained by a network of polysaccharide fibres, i.e. of water that can be regarded as a close approximation to 'biological water', vary smoothly during the transition to zero polymer concentration;

(2) gel water is less mobile than pure $\mathrm{H}_{2} \mathrm{O}$, and this is manifest in reduced self-diffusion coefficients $D_{t}$ and residence times $\tau_{\circ}$ for jump diffusion;

(3) the temperature dependence of $\tau_{0}$ suggests a lowered activation energy for translational jump diffusion relative to pure water values measured by Teixeira et al. (30);

(4) the molecular dynamics of gel water at $\mathrm{T}>270 \mathrm{~K}$ resembles that of pure supercooled water at some corresponding temperature between 250 and $270 \mathrm{~K}$, but there are some quantitative differences. A 'structural temperature' lower than the actual thermodynamic temperature can thus be assigned to gel water.

The resulting overall picture of water mobility in gels is in agreement with results of percolation models and MD simulations (31) which picture water as a continually restructuring gel-like random network of $\mathrm{H}$-bonded molecules, and predict the rapid forming and breaking of $\mathrm{H}$-bonded water clusters.

\section{INS results}

Inelastic scattering data were obtained using the time-focusing spectrometer IN6 at the ILL for a fully hydrogenous gel with concentration $C=1$ at four temperatures between 296 and 337 $\mathrm{K}$ (27). The range of energy transfers $\hbar \omega$ extends from -15 to $1000 \mathrm{~cm}^{-1}$, the scattering vectors ranging from 0.2 to $2.1 \AA^{-1}$. As in the case of QENS, the information obtained relates essentially to proton dynamics. The TOF spectra outside the quasi-elastic region $\left(\hbar \omega \geq 10 \mathrm{~cm}^{-1}\right)$ show a broad band with a maximum around $50 \mathrm{~cm}^{-1}$, which is more prominent at higher $Q$ values and is always well separated from the quasi-elastic peak. A second more prominent peak is observed at higher $\hbar \omega$ values. The latter is due to the competing effects of the increase in the scattering cross section which is $\propto Q^{2}$ at constant scattering angle and the decrease of the thermal population factors which become proportional to $\exp \left(-\hbar \omega / k_{B} T\right)$ when $\hbar \omega / k_{B} T>2-3$. The former is a feature which is also observed in other water-biopolymer assemblies as hydrated globular proteins (32).
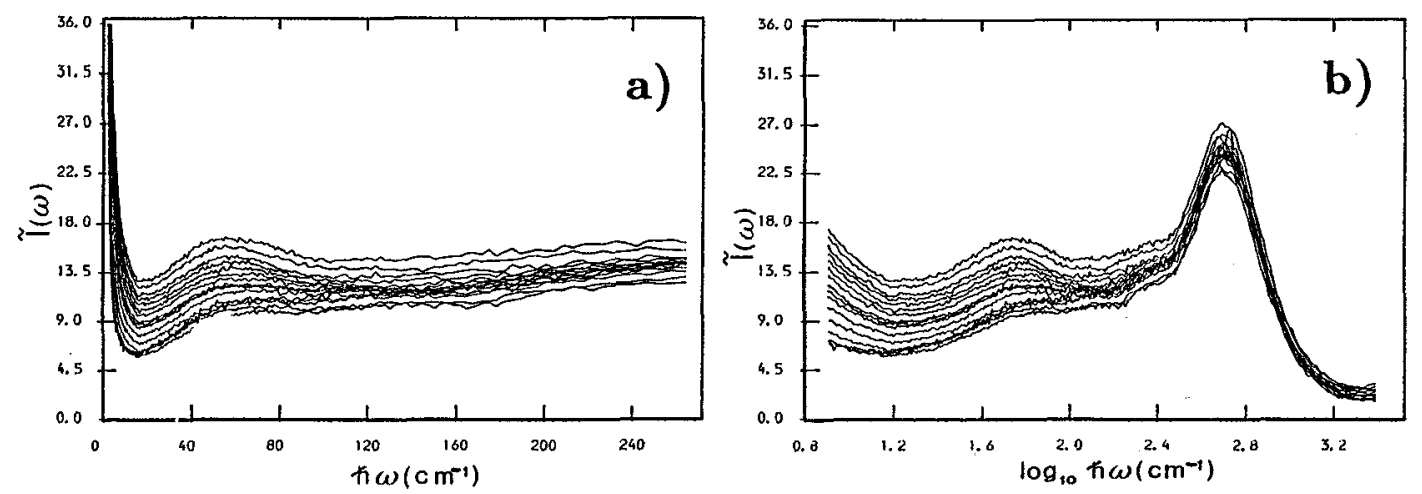

Fig. 7 TOF inelastic neutron scattering spectra at room temperature for a fully hydrogenous gel with $\mathrm{C}=1 \%$. In (a) the abscissae are linear in $\mathrm{cm}^{-1}$, while in (b) the scale is $\log _{10}$ of $\hbar \omega$ in $\mathrm{cm}^{-1}$. The range of $Q$-values covered goes from $Q=0.25 \AA^{-1}$ (lowest curve) to $Q=1.74 \AA^{-1}$ (highest curve). 
From the data shown in Fig. 7 one can derive frequency distributions $P(Q, \omega)$ which are weighted by the nuclear cross-sections, masses and squared displacement vectors. $P(Q, \omega)$ is defined as:

$$
P(Q, \omega)=\frac{6 \omega}{\hbar Q^{2}}\left(e^{\hbar \omega / k_{B} T}-1\right) S(Q, \omega)
$$

The $P(Q, \omega)$ distributions obtained from the data shown in Fig. 7 are shown in Fig. 8 .
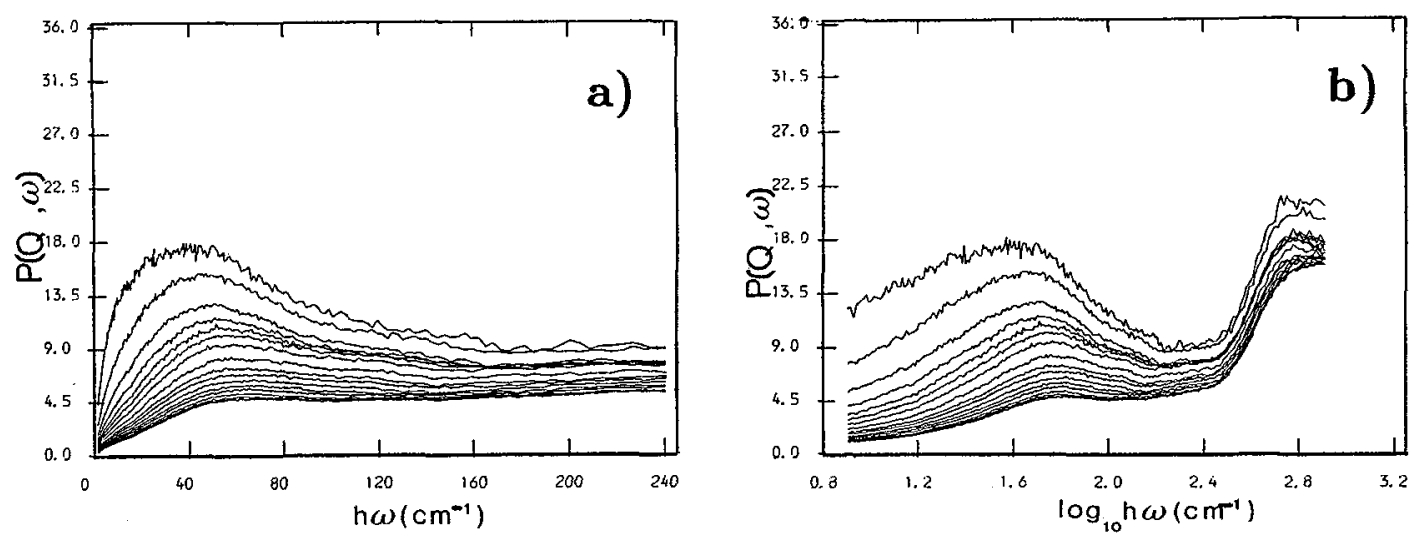

Fig. 8 Proton-amplitude weighted frequency distributions obtained from the data in Fig. 7. In (a) the abscissae are linear in $\mathrm{cm}^{-1}$, while in (b) the scale is $\log _{10}$ of $\hbar \omega$ in $\mathrm{cm}^{-1}$.

They show significant intensity increases with decreasing $Q$, i.e. with increasing scale length $2 \pi / Q$. The $Q \rightarrow 0$ limit of the $P(Q, \omega)$ curves gives a weighted density of states $G(\omega)$ characterising the vibrational dynamics of the gel. In the above mentioned studies of the dynamics of hydrated proteins $(32)$, whose $S(Q, \omega)$ curves show evident resemblances to those of our gels, deviations have been observed from the Debye model for solids in the low $\omega$ region of $G(\omega)$. They have been attributed to the presence of excitations propagating along the topologically fractal structure of the coiled polypeptide backbone. A similar interpretation could be tested for agarose gels as well. This would require the collection of a dataset of spectra with a good resolution especially in the low- $Q$ region for gel samples spanning a wide range of concentrations and temperatures.

\section{CONCLUSIONS}

We have discussed some theoretical and experimental aspects of the application of the scattering of neutrons and of Mössbauer radiation to the study of agarose gels. The diffraction results demonstrate clearly that one can obtain detailed information on the polysaccharide network over distances up to about $1000 \AA$. As concerns the molecular dynamics of gels, the use of advanced techniques as RSMR and high resolution QENS has provided much new information on the motions of the polysaccharide backbone and of the associated hydration water.

\section{REFERENCES}

/1/ Polymer Gels, Fundamentals and Biomedical Applications (De Rossi, D., Kajiwara, K., Osada, Y., Yamauchi, A. Eds.), Plenum Press N.Y., (1991).

/2/ Arnott, S., Fulmer, A., Scott, W.E., Dea, I.C.M., Moorhouse, R., Rees, D.A., J. Mol. Biol. 90 (1974) 269. 
/3/ Corongiu, G., Fornili, S.L., Clementi, E., J. Quant. Chem. 10 (1983) 277.

/4/ Mackie, W., Sellen, D.B., Sutcliffe, J., Polymer 19 (1978) 9.

/5/ Key, P.Y., Sellen, D.B., J, Polym. Sci. 20 (1982) 659.

/6/ San Biagio, P.L., Madonia, F., Sciortino, F., Palma-Vittorelli, M.B., Palma, M.U., J. Phys. Paris 45 (1984) C7:225.

/7/ Doi, M., Edwards, S.F., The Principles of Polymer Dynamics, Oxford Univ. Press, Oxford (1986).

/8/ De Gennes, P.G., Scaling Concepts in Polymer Physics, Cornell U.P., Ithaca N.Y.,(1953).

/9/ Higgins, J.S., Physica B 136 (1986) 201.

/10/ Richter, D., Physica B 180 \& 181 (1992) 7.

/11/ Anderson, N.S., Campbell, J.W., Harding, M.M., Rees, D.A., Samuel, J.W.B, J.Mol. Biol. $45(1969) 85$.

/12/ Foord, S.A., Atkins, E.D.T., Biopolymers 28 (1989) 1345.

/13/ Waki, S., Harvey, J.D., Bellamy, A.R., Biopolymers 21 (1982) 1909.

/14/ Ross-Murphy, S., in Polymer Gels, Fundamentals and Biomedical Applications (DeRossi, D., Kajiwara, K., Osada, Y., Yamauchi, A. Eds.), Plenum Press N.Y., (1991).

/15/ Flory, P.J., Principles of Polymer Chemistry, Cornell U.P., Ithaca N.Y.,(1953).

/16/ Middendorf, H.D., Cavatorta, F., Deriu, A., Progr. Colloid Polym. Sci. 81 (1990) 275.

/17/ Deriu, A., Cavatorta, F., Cabrini, D., Middendorf, H.D., Progr. Colloid Polym. Sci. 84 (1991) 461.

/18/ Kajiwara, K., Kohjiya, S., Shibayama, M., Urakawa, H., in Polymer Gels, Fundamentals and Biomedical Applications (De Rossi, D., Kajiwara, K., Osada, Y., Yamauchi, A. Eds.), Plenum Press N.Y., (1991).

/19/ Stauffer, D., Coniglio, A., Adam, M., Adv. Polym. Sci. 44 (1982) 105.

/20/ Kajiwara, K., Gordon, M., J. Chem. Phys. 59 (1973) 3623.

/21/ Perkins, S.J., Weiss, H., J. Mol. Biol. 168 (1983) 847.

/22/ Middendorf, H.D., Hotz de Baar, O. In preparation.

/23/ Franks, F., Mathias, S., The Biophysics of Water, Wiley, London (1983).

/24/ Albanese, G., Deriu, A., Rivista del Nuovo Cimento, Series 3, Vol.2, No. 9 (1979) 1.

/25/ Albanese, G., Deriu, A., Ugozzoli, F., Vignali, C., Il Nuovo Cimento D 9 (1987) 319.

/26/ Akcasu, A.Z., Benmouna, M., Han, C.C., Polymer 21 (1980) 866.

/27/ Cavatorta, F., Deriu, A., Middendorf, H.D., in Structure and Dynamics of Nucleic Acids, Proteins, And Membranes (Clementi, E., Chin, S. Eds.), Plenum, N.Y. (1986).

/28/ Middendorf, H.D., Cavatorta, F., Deriu, A., Steigenberger, U., Physica B 156 \& 157 (1989) 456.

/29/ Deriu, A., Cavatorta, F., Cabrini, D., Carlile, C.J., Middendorf, H.D., Nature (1992). Submitted.

/30/ Teixeira, J., Belissent-Funel, M.-C., Chen, S.H., Dianoux, A.J., Phys. Rev. A 31 91985) 1913.

/31/ Geiger, A., Stanley, H.E., Phys. Rev. Lett. 49 (1982); Sciortino, F., Poole, P.H., Stanley, H.E., Havlin, S., Phys. Rev. Lett. 64 (1990) 1686; Sciortino, f., Geiger, A., Stanley, H.E., Nature 354 (1991) 1121.

/32/ Smith, J.C., Quarterly Rev. Biophys. 24 (1991) 227. 